\title{
SUBSPACE-BASED SEMI-BLIND CHANNEL ESTIMATION FOR STC-OFDM
}

\author{
Yonghong Zeng and Tung Sang Ng \\ Department of Electrical and Electronic Engineering \\ The University of Hong Kong, Hong Kong
}

\begin{abstract}
A subspace-based blind method is proposed for estimating the channel responses of a STC-OFDM system with two transmit antennas and one receive antenna. It gives estimations to the two channel responses subject to two ambiguity parameters. The method is valid whether the two channel transfer functions are coprime or not and does not require precise channel order information (only an upper bound for the orders is required). Furthermore, a method is presented to resolve the ambiguities by using two or more pilot symbols. Simulations show that the methods are effective and robust.
\end{abstract}

\section{INTRODUCTION}

Combination of the space-time coding (STC) and orthogonal frequency division multiplexing (OFDM) produces the STC-OFDM which not only keeps the diversity gain of STC but also enjoys the advantages of OFDM such as multipath mitigation and fast frequency domain equalization. A distinguished example of such a combination is the STCOFDM proposed in [1], which uses two transmit antennas and one receive antenna, and achieves a diversity of order two on frequency-selective channels. Although some enhancements to STC, such as the unitary or differential STC $[2,3,4,5]$, or some detection methods, such as the maximum likelihood (ML) [6], can dispense with channel estimation in flat-fading channels at the expense of performance loss relative to coherent detection or a prohibitive high computational complexity, channel state information (CSI) is usually required for a STC system to achieve its full capacity. Hence, successful implementation of the STC over frequency selective channels greatly depends on channel estimation algorithms.

Channel estimation is usually achieved by a training method or a blind method or their combination. Training method is simple but it consumes some bandwidth which is very precious in wireless communications [7]. Blind method is usually more complex but it costs zero or very little bandwidth. Although blind methods have been studied extensively in recent years for wireless communication (see $[8,9]$ and the references herein), there have been only few works on the STC-OFDM $[1,10]$. In [1], a deterministic blind method is presented, which requires the channel transfer functions to be coprime (no common zeros) and the transmitted signals to have constant-modulus (CM). In [10], a subspace (SS) based blind method is proposed for precoded STC-OFDM. It should be noted that precoding not only increase the system complexity but also consumes additional bandwidth. In this paper, we propose a SS based method for the STC-OFDM system with two transmit antennas and one receive antenna [1]. The method does not need precoding or $\mathrm{CM}$ modulating. It gives estimations to the two channels subject to two ambiguity parameters if the zeropadding OFDM (ZP-OFDM) [11] is used and the transmitted symbol constellation is real (for example, PAM constellation). The reason of using the ZP-OFDM other than the classical cyclic-prefixed OFDM (CP-OFDM) is that the ZP-OFDM not only has all the advantages of CP-OFDM but also simplifies channel estimations $[11,9,12,13]$. The method works well even if the channel transfer functions are not coprime. To determine the two ambiguity parameters, a method which uses two or more pilot symbols is proposed. Simulations show that the methods are effective and robust.

Some notations are used in the following. Superscripts $T, \dagger$ and $*$ stand for transpose, transconjugate, and conjugate, respectively. $\mathbf{I}_{q}$ is the identity matrix of order $q$.

\section{STC-OFDM SYSTEM}

The STC-OFDM system proposed in [1] is considered here, which is shown in Figure 1. It is proved in $[1,10]$ that the system can achieve diversity of order two. Let $\mathbf{s}_{i}$ be the block symbol to be transmitted at time $i$ (before OFDM modulation), where

$$
\mathbf{s}_{i}=\left(s_{i}(0), s_{i}(1), \cdots, s_{i}(N-1)\right)^{T}, i=0,1, \cdots,
$$

and its inverse discrete Fourier transform (IDFT) is $\mathbf{u}_{i}=$ $\left(u_{i}(0), u_{i}(1), \cdots, u_{i}(N-1)\right)^{T}$. The block symbols are first encoded by a STC. After the STC, the block symbols $\mathbf{s}_{i}$ are turned to $\overline{\mathbf{s}}_{i}$ and $\tilde{\mathbf{s}}_{i}$, where $\overline{\mathbf{s}}_{2 i}=\mathbf{s}_{2 i}, \overline{\mathbf{s}}_{2 i+1}=-\mathbf{s}_{2 i+1}^{*}$, $\tilde{\mathbf{s}}_{2 i}=\mathbf{s}_{2 i+1}$ and $\tilde{\mathbf{s}}_{2 i+1}=\mathbf{s}_{2 i}^{*}$. Then two transmitters (TX1 and TX2) are used to transmit the block symbols $\overline{\mathbf{s}}_{i}$ and $\tilde{\mathbf{s}}_{i}$, respectively. At each transmitter, the block symbols are further modulated by the OFDM. We assume that zeropadding OFDM (ZP-OFDM) [11, 9] instead of cyclic prefix OFDM (CP-OFDM) is used, because ZP-OFDM avoids inter-block interference (IBI) and therefore simplifies channel estimation and equalization $[9,11,12,13]$. In ZPOFDM, a symbol block is transformed by the IDFT, and then $L(L \leq N)$ zeros are added to the tail of each transformed block (zero-padding), where cyclic prefix is no longer needed. Let $h_{j}(l)\left(l=0,1, \cdots, L_{j}\right)$ be the channel response (including the transmitting and receiving filters) from transmitter $j$ to the receiver (RX), where $L_{j}$ is the channel order, $L_{j} \leq L, j=1,2$. In the following we assume that the symbols are real numbers. Then the received length- $M$ blocks at time $2 i$ and $2 i+1$ are, respectively,

$$
\begin{aligned}
x_{2 i}(n)= & \sum_{l=0}^{L} h_{1}(l) u_{2 i}(n-l) \\
& +\sum_{l=0}^{L} h_{2}(l) u_{2 i+1}(n-l)+\eta_{2 i}(n),
\end{aligned}
$$




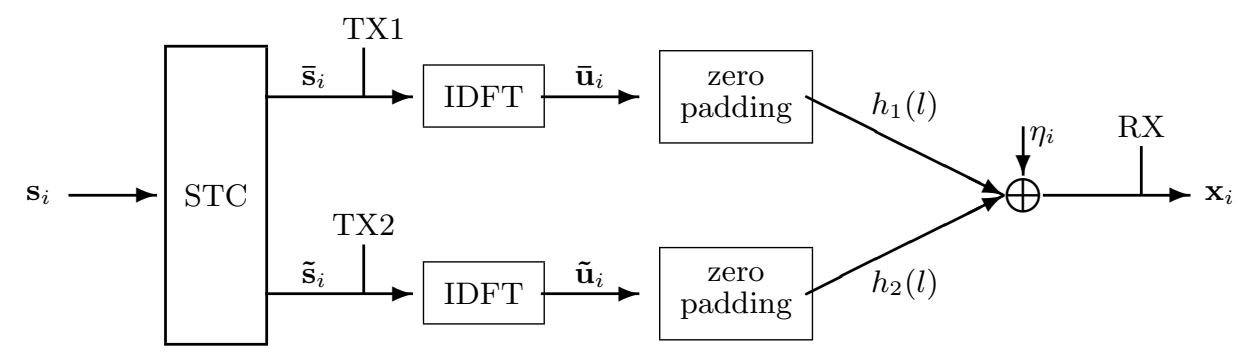

Figure 1: STC-OFDM system

$$
\begin{aligned}
x_{2 i+1}(n)= & -\sum_{l=0}^{L} h_{1}(l) u_{2 i+1}(n-l) \\
& +\sum_{l=0}^{L} h_{2}(l) u_{2 i}(n-l)+\eta_{2 i+1}(n), \\
n & =0,1, \cdots, M-1,
\end{aligned}
$$

where $M=N+L, h_{j}(l)$ is zero-padded for $L_{j}+1 \leq l \leq L$, and $\eta_{i}(n)$ is the channel noise. Note that $u_{i}(n)=0$, if $n<0$ or $N \leq n<M$.

Now let

$$
\begin{aligned}
& \mathbf{x}_{i}=\left[\begin{array}{c}
x_{i}(0) \\
x_{i}(1) \\
\vdots \\
x_{i}(M-1)
\end{array}\right], \quad \eta_{i}=\left[\begin{array}{c}
\eta_{i}(0) \\
\eta_{i}(1) \\
\vdots \\
\eta_{i}(M-1)
\end{array}\right] \\
& \mathbf{H}_{j}=\left[\begin{array}{ccccc}
h_{j}(0) & & & & \\
\vdots & h_{j}(0) & & & \\
h_{j}(L) & \vdots & \ddots & & \\
& h_{j}(L) & & \ddots & \\
& & \ddots & & h_{j}(0) \\
& & & \ddots & \vdots \\
& & & & h_{j}(L)
\end{array}\right] \text {, }
\end{aligned}
$$

where $\mathbf{H}_{1}$ and $\mathbf{H}_{2}$ are $M \times N$ lower triangular Toeplitz matrix. Then (1) and (2) can be written as

$$
\begin{aligned}
\mathbf{x}_{2 i} & =\mathbf{H}_{1} \mathbf{u}_{2 i}+\mathbf{H}_{2} \mathbf{u}_{2 i+1}+\eta_{2 i}, i=0,1, \cdots, \\
\mathbf{x}_{2 i+1} & =-\mathbf{H}_{1} \mathbf{u}_{2 i+1}+\mathbf{H}_{2} \mathbf{u}_{2 i}+\eta_{2 i+1}, i=0,1, \cdots
\end{aligned}
$$

Let

$$
\begin{aligned}
& \mathbf{r}_{i}=\left[\begin{array}{c}
\mathbf{x}_{2 i} \\
\mathbf{x}_{2 i+1}
\end{array}\right], \quad \mathbf{v}_{i}=\left[\begin{array}{c}
\mathbf{u}_{2 i} \\
\mathbf{u}_{2 i+1}
\end{array}\right], \\
& \xi_{i}=\left[\begin{array}{c}
\eta_{2 i} \\
\eta_{2 i+1}
\end{array}\right], \quad \mathbf{H}=\left[\begin{array}{cc}
\mathbf{H}_{1} & \mathbf{H}_{2} \\
\mathbf{H}_{2} & -\mathbf{H}_{1}
\end{array}\right] .
\end{aligned}
$$

Then (5) and (6) become

$$
\mathbf{r}_{i}=\mathbf{H} \mathbf{v}_{i}+\xi_{i}, i=0,1, \cdots .
$$

Another technique [14], the combination of STC and the single-carrier frequency-domain equalization, has nearly the same advantage of the STC-OFDM, but avoids OFDM's shortcomings of high peak-to-average ratio and high sensitivity to frequency errors. If the CP for each symbol block is replaced by the $\mathrm{ZP}$ as for the $\mathrm{ZP}-\mathrm{OFDM}$, the received signal in this system can be expressed as

$$
\mathbf{r}_{i}=\mathbf{H}\left[\begin{array}{c}
\mathbf{s}_{2 i} \\
\mathbf{s}_{2 i+1}
\end{array}\right]+\xi_{i}, i=0,1, \cdots
$$

Hence, a channel estimation method for a STC-OFDM is also valid for such a system.

\section{SUBSPACE-BASED CHANNEL ESTIMATION}

In this section, estimation of the channels using only the second order statistics (SOS) of the received signal samples is considered. The following statistical properties of the transmitted symbols and channel noise are assumed.

(A1) Noises are white and uncorrelated.

(A2) Noises and transmitted signals are uncorrelated.

\subsection{Identifiability}

Based on the assumptions (A1 and A2), the statistical autocorrelation matrices of $\mathbf{r}_{i}$ can be written as

$$
\mathbf{R}_{r}=\mathrm{E}\left(\mathbf{r}_{i} \mathbf{r}_{i}^{\dagger}\right)=\mathbf{H R}_{v} \mathbf{H}^{\dagger}+\sigma_{\eta}^{2} \mathbf{I}_{2 M},
$$

where $\mathbf{R}_{v}=\mathrm{E}\left(\mathbf{v}_{i} \mathbf{v}_{i}^{\dagger}\right)$ is a positive definite matrix and $\mathrm{E}(z)$ means the mathematical expectation of a random variable $z$.

The smallest eigenvalue of matrix $\mathbf{R}_{r}$ is $\sigma_{\eta}^{2}$. If $\mathbf{H}$ is of full column rank, the rank of $\mathbf{H} \mathbf{R}_{v} \mathbf{H}^{\dagger}$ is $2 N$. Thus, there are $q=2(M-N)$ co-orthogonal eigenvectors corresponding to the smallest eigenvalue. These eigenvectors are denoted by $\beta_{k}(k=0,1, \cdots, q-1)$. Based on a simple mathematical derivation which is used in the standard subspace method $[15,8,9]$, it is known that

$$
\beta_{k}^{\dagger} \mathbf{H}=0, k=0,1, \cdots, q-1,
$$

that is, $\beta_{k}(k=0,1, \cdots, q-1)$ spans the left null space of H. Having known the left null space, we can determine the range space, denoted by $\operatorname{span}(\mathbf{H})$, which is all the possible linear combinations of the column vectors of $\mathbf{H}$. Based on the special structure of $\mathbf{H}$, in the following we will prove that $\mathbf{H}$ is uniquely determined by $\operatorname{span}(\mathbf{H})$ subject to two ambiguity parameters.

Lemma 1 There exist permutation matrices $\mathbf{P}$ and $\mathbf{Q}$ such that

$$
\mathbf{P H Q}=\mathbf{F},
$$

where

$$
\mathbf{F}=\left[\begin{array}{ccccc}
\mathbf{F}(0) & & & & \\
\vdots & \mathbf{F}(0) & & & \\
\mathbf{F}(L) & \vdots & \ddots & & \\
& \mathbf{F}(L) & & \ddots & \\
& & \ddots & & \mathbf{F}(0) \\
& & & \ddots & \vdots \\
& & & & \mathbf{F}(L)
\end{array}\right]
$$




$$
\mathbf{F}(l)=\left[\begin{array}{cc}
h_{1}(l) & h_{2}(l) \\
h_{2}(l) & -h_{1}(l)
\end{array}\right] .
$$

Lemma 2 If $h_{1}^{2}(0)+h_{2}^{2}(0) \neq 0, \mathbf{F}$ is of full column rank.

It is easy to prove the two lemmas. A more general result of Lemma 2 is also proved in [13].

Theorem 1 Let $\mathbf{H}_{j}$ and $\mathbf{H}$ be defined as before. $\hat{\mathbf{H}}_{j}$ and $\hat{\mathbf{H}}$ are defined similarly as $\mathbf{H}_{j}$ and $\mathbf{H}$, respectively, with $h_{j}(l)$ replaced by $\hat{h}_{j}(l)(j=1,2)$. Assume that $h_{1}^{2}(0)+h_{2}^{2}(0) \neq 0$ and $\hat{h}_{1}^{2}(0)+\hat{h}_{2}^{2}(0) \neq 0$. If $\operatorname{span}(\mathbf{H})=\operatorname{span}(\hat{\mathbf{H}})$, there exist two constants $b_{1}$ and $b_{2}$ such that

$$
\begin{aligned}
& \hat{h}_{1}(l)=b_{1} h_{1}(l)-b_{2} h_{2}(l), \\
& \hat{h}_{2}(l)=b_{2} h_{1}(l)+b_{1} h_{2}(l), l=0,1, \cdots, L .
\end{aligned}
$$

Proof. It is easy to verify that $\operatorname{span}(\mathbf{H})=\operatorname{span}(\hat{\mathbf{H}})$ if and only if there exists an $2 N \times 2 N$ invertible matrix $\mathbf{B}$ such that $\hat{\mathbf{H}}=\mathbf{H B}$. By defining a matrix $\hat{\mathbf{F}}$ similar to $\mathbf{F}$ with $h_{j}(l)$ $(j=1,2)$ replaced by $\hat{h}_{j}(l)$, it is obvious that $\mathbf{P} \hat{\mathbf{H}} \mathbf{Q}=\hat{\mathbf{F}}$. So,

$$
\mathbf{P}^{T} \hat{\mathbf{F}} \mathbf{Q}^{T}=\mathbf{P}^{T} \mathbf{F} \mathbf{Q}^{T} \mathbf{B} .
$$

Therefore,

$$
\hat{\mathbf{F}}=\mathbf{F} \mathbf{Q}^{T} \mathbf{B Q} .
$$

Since $\mathbf{Q}^{T} \mathbf{B Q}$ is an invertible matrix, it follows that $\operatorname{span}(\mathbf{F})=\operatorname{span}(\hat{\mathbf{F}})$. Based on the assumptions of the theorem and Lemma 2, $\mathbf{F}$ and $\hat{\mathbf{F}}$ are of full column rank. Therefore, from the Theorem 1 in [13], there exists an $2 \times 2$ invertible matrix $\mathbf{b}$ such that

$$
\hat{\mathbf{F}}(l)=\mathbf{F}(l) \mathbf{b}, \quad l=0,1, \cdots, L,
$$

where $\mathbf{F}(l)$ is defined in $(13)$ and $\hat{\mathbf{F}}(l)$ is defined similarly with $h_{j}(l)$ replaced by $\hat{h}_{j}(l)$. Let

$$
\mathbf{b}=\left[\begin{array}{ll}
b_{1} & b_{2} \\
b_{3} & b_{4}
\end{array}\right]
$$

From (15), it follows that

$$
\begin{aligned}
& {\left[\begin{array}{cc}
h_{1}(l) & h_{2}(l) \\
h_{2}(l) & -h_{1}(l)
\end{array}\right]\left[\begin{array}{cc}
\hat{h}_{1}(l) & \hat{h}_{2}(l) \\
\hat{h}_{2}(l) & -\hat{h}_{1}(l)
\end{array}\right]} \\
& =\left[\begin{array}{cc}
h_{1}^{2}(l)+h_{2}^{2}(l) & 0 \\
0 & h_{1}^{2}(l)+h_{2}^{2}(l)
\end{array}\right]\left[\begin{array}{ll}
b_{1} & b_{2} \\
b_{3} & b_{4}
\end{array}\right] .
\end{aligned}
$$

From the above equation, it is easy to show that $b_{4}=b_{1}$ and $b_{3}=-b_{2}$. Thus (14) is easily obtained and proof of the theorem is complete.

From this theorem, it is apparent that, using the SS method, the channels are identifiable with two ambiguity parameters when only an upper bound is given for the channel orders.

\subsection{Implementation of the method}

Equation (11) can be expressed equivalently as

$$
\mathbf{H}^{\dagger} \beta_{k}=0, k=0,1, \cdots, q-1 .
$$

Since $\mathbf{H}=\mathbf{P}^{T} \mathbf{F} \mathbf{Q}^{T}$, we have $\mathbf{F}^{\dagger} \mathbf{P} \beta_{k}=0, k=0,1, \cdots, q-1$. For simplicity, let $\alpha_{k}=\mathbf{P} \beta_{k}$. By dividing the vector $\alpha_{k}$ into blocks as $\alpha_{k}=\left[\alpha_{k}^{T}(M-1), \alpha_{k}^{T}(M-2), \cdots, \alpha_{k}^{T}(0)\right]^{T}$, where $\alpha_{k}(m)(m=0,1, \cdots, M-1)$ are $2 \times 1$ vectors, it is easy to turn (16) into

$$
\sum_{l=0}^{L} \alpha_{k}^{\dagger}(n+L-l) \mathbf{F}(l)=0, n=0,1, \cdots, N-1 .
$$

Denote two matrices $\mathbf{G}_{k}$ and $\overline{\mathbf{F}}$ as

$$
\begin{gathered}
\mathbf{G}_{k}=\left[\begin{array}{ccccc}
\alpha_{k}^{\dagger}(L) & \alpha_{k}^{\dagger}(L-1) & \cdots & \cdots & \alpha_{k}^{\dagger}(0) \\
\alpha_{k}^{\dagger}(L+1) & \alpha_{k}^{\dagger}(L) & \cdots & \cdots & \alpha_{k}^{\dagger}(1) \\
\vdots & \vdots & & & \vdots \\
\alpha_{k}^{\dagger}(M-1) & \alpha_{k}^{\dagger}(M-2) & \cdots & \cdots & \alpha_{k}^{\dagger}(N-1)
\end{array}\right] \\
\overline{\mathbf{F}}=\left[\begin{array}{llll}
\mathbf{F}^{T}(0) & \mathbf{F}^{T}(1) & \cdots & \mathbf{F}^{T}(L)
\end{array}\right]^{T} .
\end{gathered}
$$

Then (17) is equivalent to

$$
\mathbf{G}_{k} \overline{\mathbf{F}}=0, k=0,1, \cdots, q-1 .
$$

(18) can also be expressed as

$$
\mathbf{G} \overline{\mathbf{F}}=0,
$$

where $\mathbf{G}$ is a $q N \times 2(L+1)$ matrix defined as $\mathbf{G}=\left[\begin{array}{llll}\mathbf{G}_{0}^{T} & \mathbf{G}_{1}^{T} & \cdots & \mathbf{G}_{q-1}^{T}\end{array}\right]^{T}$. We use $\overline{\mathbf{F}}(:, 1)$ (Matlab notation) to denote the first column of the matrix $\overline{\mathbf{F}}$. Then

$$
\mathbf{G} \overline{\mathbf{F}}(:, 1)=0 \text {. }
$$

Thus, $\overline{\mathbf{F}}(:, 1)$ belongs to the right null space of $\mathbf{G}$. From Theorem 1, it is easy to show that the dimension of right null space of $\mathbf{G}$ is 2 . Let $\mathbf{w}_{1}$ and $\mathbf{w}_{2}$ be a basis of the null space. Then, the channels can be expressed as

$$
\overline{\mathbf{F}}(:, 1)=c_{1} \mathbf{w}_{1}+c_{2} \mathbf{w}_{2},
$$

where $c_{1}$ and $c_{2}$ are two parameters to be determined.

The subspace method is summarized as follows.

Algorithm 1 Subspace-based Channel Estimation for STCOFDM

Step 1. Compute $\mathbf{R}_{r}=\mathrm{E}\left(\mathbf{r}_{i} \mathbf{r}_{i}^{\dagger}\right)$.

Step 2. Find $q=2(M-N)$ co-orthogonal eigenvectors, $\beta_{k}(k=0,1, \cdots, q-1)$, corresponding to the least $q$ eigenvalues of matrix $\mathbf{R}_{r}$.

Step 3. Form the matrix $\mathbf{G}$ defined above from $\beta_{k}$ and compute the SVD of $\mathbf{G}$. Choose a right singular vector, denoted by $\mathbf{w}=[w(0), w(1), \cdots, w(2 L-1)]^{T}$, corresponding to the least singular value. Then the channels are $h_{1}(l)=$ $b_{1} w(2 l)-b_{2} w(2 l+1), h_{2}(l)=b_{2} w(2 l)+b_{1} w(2 l+1), l=$ $0,1, \cdots, L$, where $b_{1}$ and $b_{2}$ are two constants which cannot be determined by the SS method.

\section{RESOLVE THE AMBIGUITY}

Given an estimation of the channels with ambiguities from the SS method, say, $\tilde{h}_{1}(l)$ and $\tilde{h}_{2}(l)$, the real channels are then $h_{1}(l)=b_{1} \tilde{h}_{1}(l)-b_{2} \tilde{h}_{2}(l), h_{2}(l)=b_{2} \tilde{h}_{1}(l)+b_{1} \tilde{h}_{2}(l)$, where $b_{1}$ and $b_{2}$ are two parameters to be determined. To determine the two parameters, we can use pilot inputs. Assume that $s_{2 i}(n)$ and $s_{2 i+1}(n)\left(n=0,1, \cdots, N_{p}-1\right)$ are pilot symbols for a fixed $i$. $b_{1}$ and $b_{2}$ can be resolved by using these pilots. Let

$$
\hat{x}_{i}(n)=\left\{\begin{array}{ll}
x_{i}(n)+x_{i}(n+N), & n=0,1, \cdots, L-1 \\
x_{i}(n), & n=L, \cdots, N-1
\end{array},\right.
$$


and $y_{i}(n)$ be the DFT of $\hat{x}_{i}(n)(n=0,1, \cdots, N-1)$. Zeropad each channel $h_{j}(l)$ to length- $N$ and let the DFT (length$N$ ) of the channels be $g_{j}(n)$. Then it is easy to prove that

$$
\begin{aligned}
y_{2 i}(n) & =g_{1}(n) s_{2 i}(n)+g_{2}(n) s_{2 i+1}(n)+\zeta_{2 i}(n), \\
y_{2 i+1}(n) & =g_{2}(n) s_{2 i}(n)-g_{1}(n) s_{2 i+1}(n)+\zeta_{2 i+1}(n),
\end{aligned}
$$

where $\zeta_{i}(n)$ is the noise after the DFT. By defining $\tilde{g}_{1}(n)$ and $\tilde{g}_{2}(n)$ to be the length- $N$ DFTs of $\tilde{h}_{1}(n)$ and $\tilde{h}_{2}(n)$, respectively, we obtain

$$
\begin{aligned}
& {\left[\begin{array}{cc}
\lambda_{1}(0) & \lambda_{2}(0) \\
-\lambda_{2}(0) & \lambda_{1}(0) \\
\vdots & \vdots \\
\lambda_{1}\left(N_{p}-1\right) & \lambda_{2}\left(N_{p}-1\right) \\
-\lambda_{2}\left(N_{p}-1\right) & \lambda_{1}\left(N_{p}-1\right)
\end{array}\right]\left[\begin{array}{c}
b_{1} \\
b_{2}
\end{array}\right]} \\
& =\left[\begin{array}{c}
y_{2 i}(0) \\
y_{2 i+1}(0) \\
\vdots \\
y_{2 i}\left(N_{p}-1\right) \\
y_{2 i+1}\left(N_{p}-1\right)
\end{array}\right]+\left[\begin{array}{c}
\zeta_{2 i}(0) \\
\zeta_{2 i+1}(0) \\
\vdots \\
\zeta_{2 i}\left(N_{p}-1\right) \\
\zeta_{2 i+1}\left(N_{p}-1\right)
\end{array}\right]
\end{aligned}
$$

where $\lambda_{1}(n)=\tilde{g}_{1}(n) s_{2 i}(n)+\tilde{g}_{2}(n) s_{2 i+1}(n)$ and $\lambda_{2}(n)=$ $-\tilde{g}_{2}(n) s_{2 i}(n)+\tilde{g}_{1}(n) s_{2 i+1}(n)$. It is easy to obtain a LS estimation for $b_{1}$ and $b_{2}$ from (22). Especially, when $N_{p}=1$, that is, only two pilot symbols are used, the estimation is

$$
\begin{aligned}
b_{1} & =\frac{1}{\lambda_{1}^{2}(0)+\lambda_{2}^{2}(0)}\left[\lambda_{1}(0) y_{2 i}(0)-\lambda_{2}(0) y_{2 i+1}(0)\right] \\
b_{2} & =\frac{1}{\lambda_{1}^{2}(0)+\lambda_{2}^{2}(0)}\left[\lambda_{2}(0) y_{2 i}(0)+\lambda_{1}(0) y_{2 i+1}(0)\right]
\end{aligned}
$$

\section{SIMULATIONS AND DISCUSSIONS}

The auto-correlation matrix $\mathbf{R}_{r}$ is approximated by $\mathbf{R}_{r}=$ $\frac{1}{N_{s}} \sum_{i=1}^{N_{s}} \mathbf{r}_{i} \mathbf{r}_{i}^{\dagger}$, where $N_{s}$ is the number of block samples used. As usual, the signal-noise-ratio (SNR) means the ratio of the average received signal power to the average noise power $\mathrm{SNR}=\mathrm{E}\left(\left|x_{i}(n)-\eta_{i}(n)\right|^{2}\right) / \mathrm{E}\left(\left|\eta_{i}(n)\right|^{2}\right)$. The normalized mean square error (NMSE) between the estimated and true channel responses is defined as

$$
\mathrm{NMSE}=\frac{\sum_{j=1}^{2} \sum_{l=0}^{L}\left|\hat{h}_{j}(l)-h_{j}(l)\right|^{2}}{\sum_{j=1}^{2} \sum_{l=0}^{L}\left|h_{j}(l)\right|^{2}},
$$

where $\hat{h}_{j}(l)$ and $h_{j}(l)$ are the estimated and true channel responses respectively.

Since the determination of the ambiguity parameters is unrelated to the SS method, it is logical to use the best estimation of the parameters to compute the NMSE for evaluating the SS method. The best parameters $b_{1}$ and $b_{2}$ can be obtained from optimization using the true channels. Using such a means to get the ambiguity parameters is of course not practical, however, it best describes the performance of the SS method. So, in the following, two NMSE curves are usually given, one corresponds to the ambiguities resolved by the pilot-based method and the other by optimization. Extensive simulations have been done to verify the effectiveness of the method. Some examples are given below. In the examples, the system parameters are as follows. The transmitted baseband signals are BPSK and the length of each OFDM block is $N=32$, which is zero-padded to a block of length $M=41$. The channels are generated randomly with the true channel orders being 4 and 7 , respectively (the two

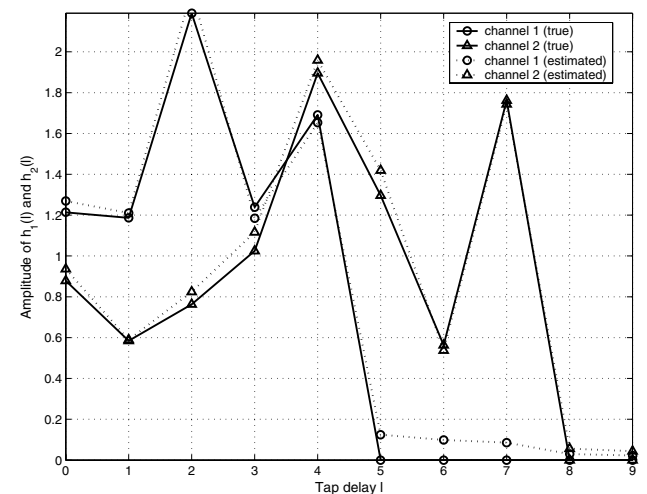

Figure 2: True and estimated channels $\left(\mathrm{SNR}=25 \mathrm{~dB}, N_{p}=1\right.$ and $\left.N_{s}=90\right)$

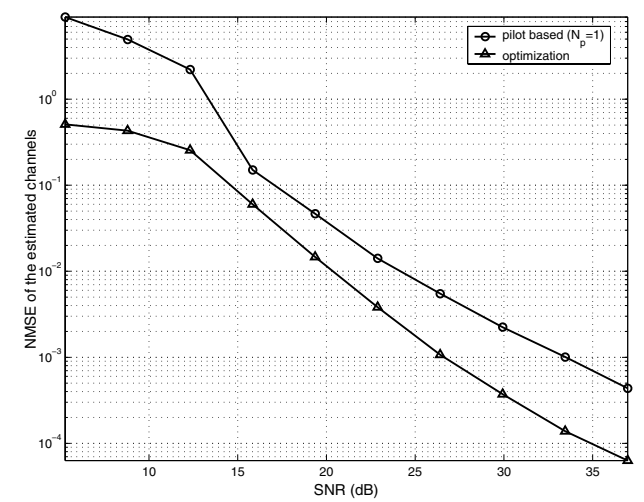

Figure 3: NMSE versus $\operatorname{SNR}\left(N_{s}=90\right)$

channels have different channel orders and 9 is an upper bound for them). The amplitudes of the two channels are shown in Figure 2. All results below are averaged over 100 Monte Carlo realizations on inputs and noises.

EXAMPLE 1. (NMSE versus SNR) When $N_{s}=90$ and $N_{p}=1$ (the number of pilot symbols is $2 N_{p}$ ), the NMSEs are shown in Figure 3 with SNR varying from $5 \mathrm{~dB}$ to $35 \mathrm{~dB}$. From the figure, we see that the semi-blind method works well. However, it also shows that the pilot-based method usually cannot get the best values for the ambiguity parameters, which means that there is a room to improve the pilot-based method for resolving the ambiguities.

EXAMPLE 2. (NMSE versus pilot size) When $S N R=$ $25 d B$ and $N_{s}=90$, the NMSE is shown in Figure 4 with $N_{p}$ varying from 1 to 9 . It is seen that the NMSE generally decrease with the increase of the number of pilots.

EXAMPLE 3. (NMSE versus SNR, not coprime channels) Here we consider the situation when the two channels are not coprime. The two transfer functions are $h_{1}(z)=$ $\left(z^{-1}-\sqrt{2}-\sqrt{2} i\right)\left[0.8644+0.3962 i+(0.0942-0.9649 i) z^{-1}-\right.$ $(0.8519-0.1684 i) z^{-2}+(0.8735-1.9654 i) z^{-3}-(0.4380+$ $\left.0.7443 i) z^{-4}-(0.4297+0.5523 i) z^{-5}-(1.1027+0.8197 i) z^{-6}\right]$ and $h_{2}(z)=\left(z^{-1}-\sqrt{2}-\sqrt{2} i\right)[1.1091-0.8661 i-(0.6149+$ $2.1165 i) z^{-1}-(0.2546+0.9645 i) z^{-2}-(0.2698-0.2127 i) z^{-3}-$ $(1.6720-0.4779 i) z^{-4}-(1.8760-0.1007 i) z^{-5}+(0.5750+$ $\left.0.2974 i) z^{-6}\right]$, respectively. Other conditions are the same as those in Example 1. Figure 5 shows the NMSEs versus the SNR. It is seen that the SS method itself still works very well, but the pilot-based method for determining the ambiguities is somewhat worsen. 


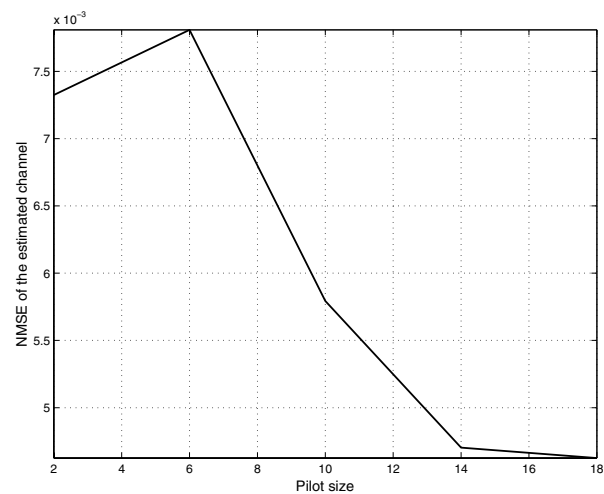

Figure 4: NMSE versus pilot size $\left(\mathrm{SNR}=25 \mathrm{~dB}, N_{s}=90\right)$



Figure 5: NMSE versus SNR (not coprime channels)

The proposed method inherits some advantages of the SS technique, such as simple structure and good performance. Sensitive to order overestimation is a major drawback of some known SS methods in wireless communication $[15,8,9]$, but here this drawback has been overcome (only an upper bound for the channel orders is required for the proposed method) due to the special structure of STC and ZP-OFDM. Compared to the method in [1], the proposed one eliminates two constraints: coprime transfer functions and constant modulus transmit signals. Compared to the method in [10], no precoding is needed in the proposed method and therefore bandwidth is saved and system complexity is reduced. However, the method does have a drawback: it requires the input symbol constellation to be real. When the input is complex, equations (1) and (2) are changed. We have not found any theoretical proof for the identifiability of the SS based method which can deal with complex inputs.

\section{CONCLUSIONS}

A subspace-based blind method has been proposed for estimating the channel responses of a STC-OFDM system. It gives estimations to all channel responses subject to two ambiguity parameters. Unlike some subspace methods in wireless communications, the proposed method does not need precise channel order information (only requires an upper bound for the orders) and does not subject to the constraint of channel coprime. Furthermore, a method has been proposed to resolve the ambiguities by using two or more pilot symbols. Simulations have shown that the methods are effective and robust.

\section{REFERENCES}

[1] Z. Liu, G. B. Giannakis, S. Barbarossa, and A. Scaglione, "Transmit antennae space-time block coding for generalized OFDM in the presence of unknown multipath," IEEE J. Select. Areas Commun., vol. 19, no. 7, pp. 1352-1364, 2001.

[2] B. M. Hochwald and T. L. Marzetta, "Unitary spacetime modulation for multiple-antenna communications in rayleigh flat fading," IEEE Trans. Inform. Theory, vol. 46, pp. 543-564, 2000.

[3] B. M. Hochwald and W. Sweldens, "Differential unitary space-time modulation," IEEE Trans. Communications, vol. 48, pp. 2041-2052, Dec. 2000.

[4] B. L. Hughes, "Differential space-time modulation," IEEE Trans. Inform. Theory, vol. 46, pp. 2567-2578, Nov. 2000.

[5] V. Tarokh and H. Jafarkhani, "A differential detection scheme for transmit diversity," IEEE J. Select. Areas Commun., vol. 18, pp. 1169-1174, July 2000.

[6] E. G. Larsson, P. Stoica, and J. Li, "Orthogonal space-time block codes: maximum likelihood detection for unknown channels and unstructured interferences," IEEE Trans. Signal Processing, vol. 51, no. 2, pp. 362$372,2003$.

[7] C. Budianu and L. Tong, "Channel estimation for space-time orthogonal block codes," IEEE Trans. Signal Processing, vol. 50, no. 10, pp. 2515-2528, 2002.

[8] Z. Ding and Y. Li, Blind Equalization and Identification. New York: Marcel Dekker, Inc., 2001.

[9] G. B. Giannakis, Y. Hua, P. Stoica, and L. Tong, Signal Processing Advances in Wireless \& Mobile Communications, vol. 1. Prentice Hall PTR, 2001.

[10] S. Zhou, B. Muquet, and G. B. Giannakis, "Subspacebased (semi-) blind channel estimation for block precoded space-time OFDM," IEEE Trans. Signal Processing, vol. 50, no. 5, pp. 1215-1228, 2002.

[11] A. Scaglione, G. B. Giannakis, and S. Barbarossa, "Redundant filterbank precoders and equalizers-part ii: blind channel estimation, synchronization and direct equalization," IEEE Trans. Signal Processing, vol. 47, no. 7, pp. 2007-2022, 1999.

[12] B. Muquet, Z. Wang, G. B. Giannakis, M. D. Courville, and P. Duhamel, "Cyclic prefixing or zero padding for wireless multicarrier transmissions," IEEE Trans. Communications, vol. 50, no. 12, pp. 2136-2148, 2002.

[13] Y. H. Zeng and T. S. Ng, "A semi-blind channel estimation method for multi-user multi-antenna OFDM systems," IEEE Trans. Signal Processing, vol. 52, no. $5,2004$.

[14] N. Al-Dhahir, "Single-carrier frequency-domain equalization for space-time block-coded transmissions over frequency-selective fading channels," IEEE Communication Letters, pp. 304-306, July 2001.

[15] E. Moulines, P. Duhamel, J. F. Cardoso, and S. Mayrargue, "Subspace methods for the blind identification of multichannel FIR filters," IEEE Trans. Signal Processing, vol. 43, no. 2, pp. 516-525, 1995. 